\title{
Facile Synthesis of Multi-channel Surface Modified Amorphous Iron Oxide Nanospheres as a High- performance Anode Material for Lithium-ion Batteries
}

\section{Shijin Yu}

Jingdezhen Ceramic Institute https://orcid.org/0000-0003-3842-5015

Wenzhen Zhu

Jingdezhen Ceramic Institute

Zhuohao Xiao

Jingdezhen Ceramic Institute

Jiahao Tong

Jingdezhen Ceramic Institute

Quanya Wei

Jingdezhen Ceramic Institute

Tianrui Chen

Jingdezhen Ceramic Institute

Xuannan He

Jingdezhen Ceramic Institute

Hua Zhu

Jingdezhen Ceramic Institute

Qiuyun Fu ( $\square$ fuqy@mail.hust.edu.cn )

LingBing Kong

Shenzhen Technology University

Fajun Wang

Jiangsu University of Technology

\section{Research Article}

Keywords: Lithium ion battery, Fe203, Amorphous, Anode, Diffusion coefficient

Posted Date: December 8th, 2021

DOI: https://doi.org/10.21203/rs.3.rs-1130829/v1 
License: (c) (i) This work is licensed under a Creative Commons Attribution 4.0 International License. Read Full License 


\section{Abstract}

The application of iron oxide as anode of lithium-ion batteries is hindered by its poor cycle stability, low rate performance and large voltage hysteresis. To address these problems, multi-channel surface modified amorphous $\mathrm{Fe}_{2} \mathrm{O}_{3}$ nanospheres were synthesized by using a facile hydrothermal method, which exhibited outstanding electrochemical performances. According to crystalline state and microstructure, it was found that surface structure of the amorphous $\mathrm{Fe}_{2} \mathrm{O}_{3}$ nanospheres can be controlled by adjusting the reaction time, due to the synergistic effect of ripening and hydrogen ion etching. Owing to the isotropic nature and the absence of grain boundaries, the amorphous $\mathrm{Fe}_{2} \mathrm{O}_{3}$ nanospheres could withstand high strains during the intercalation of lithium ions. Meanwhile, the multi-channel surface structure can not only increase the contact area between $\mathrm{Fe}_{2} \mathrm{O}_{3}$ nanospheres and electrolyte, but also reserve space for volume expansion after lithium storage, thereby effectively alleviating the volume change during the intercalation-deintercalation of lithium ions. As confirmed by the Galvanostatic intermittent titration analysis results, the amorphous $\mathrm{Fe}_{2} \mathrm{O}_{3}$ electrode had higher $\mathrm{Li}^{+}$diffusion coefficient than the crystalline counterpart. As a result, the multi-channel surface modified amorphous $\mathrm{Fe}_{2} \mathrm{O}_{3}$ electrode exhibited higher specific capacity, more stable cycle performance and narrower voltage hysteresis. It is believed that amorphous metal oxides have great potential as high-performance anode of next-generation lithium-ion batteries.

\section{Introduction}

Due to their high energy/power densities and long cycle life, lithium ion batteries (LIBs) have been widely used in portable electronic devices and electric vehicles (EV) [1]. However, the commercial graphite electrodes with low theoretical capacity of $372 \mathrm{mAh} / \mathrm{g}$ cannot meet the requirements of high energy density $[2,3]$. Therefore, various anode materials with higher specific capacity than graphite have been explored, such as metal [4], silicon-based materials [5, 6], MXene [7], nitrides [8, 9], sulfides [10] and transition metal oxides (TMOs) [11]. Iron oxide $\left(\mathrm{Fe}_{2} \mathrm{O}_{3}\right)$ has received widespread attention, due to its natural abundance, low price, non-toxicity and high theoretical specific capacity $(1007 \mathrm{mAh} / \mathrm{g})[12,13]$. Unfortunately, similar to other $\mathrm{TMOs}, \mathrm{Fe}_{2} \mathrm{O}_{3}$ also has poor electronic conductivity, low Coulomb efficiency, high potential hysteresis and large volume change, during the charge/discharge process, leading to rapid fading in capacity $[14,15]$.

In order to tackle these issues of $\mathrm{Fe}_{2} \mathrm{O}_{3}$ anodes, various strategies have been proposed recently. For instance, one strategy is to build 1D-3D nanostructures, such as nanowires [16], nanorods [17], nanotubes [18], nanosheets [19], nanoboxes [20], flower-like [21] and nanospheres [22]. Gu et al. used $\mathrm{FeC}_{2} \mathrm{O}_{4} \cdot 2 \mathrm{H}_{2} \mathrm{O}$ nanorods as a sacrificial template to synthesize hollow $\mathrm{Fe}_{2} \mathrm{O}_{3}$ nanotubes, the $\mathrm{Fe}_{2} \mathrm{O}_{3}$ electrode had a specific capacity of $764.2 \mathrm{mAh} / \mathrm{g}$ after 500 cycles at a current density of $0.5 \mathrm{~A} / \mathrm{g}[18]$. With these $\mathrm{Fe}_{2} \mathrm{O}_{3}$ nanostructures, the diffusion path of lithium ions can be effectively shortened and the drastic volume changes during the lithiation/delithiation process can be alleviated. Another way is to hybridize $\mathrm{Fe}_{2} \mathrm{O}_{3}$ with conductive carbonaceous materials or metals, thereby increasing the conductivity and the diffusion 
rate of lithium ions. Yu et al. reported $\mathrm{Fe}_{2} \mathrm{O}_{3} /$ graphene hybrid-based electrodes with largely enhanced conductivity, a high reversible capacity of $658.5 \mathrm{~mA} \mathrm{~h} / \mathrm{g}$ was achieved after 200 cycles at $1 \mathrm{~A} / \mathrm{g}$ [23]. Finally, $\mathrm{Fe}_{2} \mathrm{O}_{3}$ has also been compounded with other metal oxides to achieve high electrochemical performances. In a binary hybrid, the two active materials reacted with lithium at different voltages, respectively. The synergistic effect between them can not only improve the storage performance of lithium, but also inhibit the expansion of electrode materials [24]. Zhang et al. prepared $\mathrm{Fe}_{2} \mathrm{O}_{3} / \mathrm{SnSSe}$ hexagonal nanoplates from hot-inject process in oil phase, the hybrid anode can maintain a capacity of $755 \mathrm{mAh} / \mathrm{g}$ after 100 cycles at a current density of $200 \mathrm{~mA} / \mathrm{g}[25]$. However, these methods are either complicated operations or involve organic solvents, leading to electrode materials with high costs.

In contrast to the intercalation reaction of graphite electrodes, transition metal oxide (TMO) can interact with lithium through phase conversion reaction $\left(\mathrm{MO}_{x}+2 x \mathrm{Li} \leftrightarrow \mathrm{M}+\mathrm{xLi}_{2} \mathrm{O}\right)$. Therefore, the feasibility and reversibility of the reaction process are dependent on the thermodynamic and kinetic parameters of the conversion reaction. The Gibbs free energy change $(\Delta G)$ of the reaction between amorphous $\mathrm{Fe}_{2} \mathrm{O}_{3}$ and lithium is $0.27 \mathrm{eV}$ lower than that of its crystalline counterpart. The lower the Gibbs free energy change, the stronger the reversibility of the conversion reaction [26]. Shi and Zhu combined amorphous $\mathrm{Fe}_{2} \mathrm{O}_{3}$ with graphene or nitrogen-doped carbon, respectively, and the corresponding electrodes were used in lithium-ion batteries or sodium-ion batteries to achieve high cycle stability [27, 28]. In addition, amorphous materials have higher capability to withstand strains and shorter pathway for the diffusion of lithium ions [29-31]. It has been confirmed that anodes based on amorphous phases $\mathrm{Si}, \mathrm{Fe}_{2} \mathrm{O}_{3}, \mathrm{TiO}_{2}$ and $\mathrm{SnO}_{2}$ could buffer the volume change and hence exhibited improved cycle performance [32-36]. Therefore, it is expected that amorphous materials are promising candidates as electrode, due to their fast reaction kinetics, strong reversibility and narrowe potential hysteresis [37, 38].

In this work, a simple and low-cost hydrothermal method was used to synthesize multi-channel surface modified amorphous $\mathrm{Fe}_{2} \mathrm{O}_{3}$ nanospheres as LIBs anodes. Besides the isotropic nature and the lack of grain boundaries, the multi-channel structure is more favorable for the intercalation and deintercalation of lithium ions, and ensures intimate contact between the active materials and electrolyte, which is very beneficial to the cycle stability of the electrode. Compared with its crystalline counterpart, the multichannel surface modified amorphous $\mathrm{Fe}_{2} \mathrm{O}_{3}$ electrode exhibits higher specific capacity, more stable cycle performance and narrower voltage hysteresis.

\section{Experimental Section}

\subsection{Materials}

Potassium hexacyanoferrate $\left(\mathrm{K}_{3}\left[\mathrm{Fe}(\mathrm{CN})_{6}\right]\right)$, ammonium dihydrogen phosphate $\left(\mathrm{NH}_{4} \mathrm{H}_{2} \mathrm{PO}_{4}\right)$, ferric chloride hexahydrate $\left(\mathrm{FeCl}_{3} \cdot 6 \mathrm{H}_{2} \mathrm{O}\right)$ and nickel acetate tetrahydrate $\left(\mathrm{Ni}\left(\mathrm{CH}_{3} \mathrm{COO}\right)_{2} \cdot 4 \mathrm{H}_{2} \mathrm{O}\right)$ were all analytically pure and supplied by China Sinopharm Chemical Reagent Co., Ltd. Analytically pure ethanol 
was provided by Tianjin Yufutai Chemical Reagent Co., Ltd. Deionized water was made in the laboratory. All reagents were not further processed.

\subsection{Synthesis of multi-channel surface modified amorphous $\mathrm{Fe}_{2} \mathrm{O}_{3}$ nanospheres}

Multi-channel surface modified amorphous $\mathrm{Fe}_{2} \mathrm{O}_{3}$ nanospheres were prepared by using a simple and lowcost hydrothermal method. $0.326 \mathrm{~g} \mathrm{~K}_{3}\left[\mathrm{Fe}(\mathrm{CN})_{6}\right]$ and $0.043 \mathrm{~g} \mathrm{NH}_{4} \mathrm{H}_{2} \mathrm{PO}_{4}$ were dissolved deionized water to form a clear solution after stirring at room temperature for $30 \mathrm{~min}$. Then, the solution was transferred to a hydrothermal kettle for hydrothermal treatment at $200^{\circ} \mathrm{C}$ for different times $(1 \mathrm{~h}, 3 \mathrm{~h}$ and $6 \mathrm{~h})$, with the corresponding samples to be denoted as A1h, A3h and A6h, respectively. The products were centrifuged and washed for three times with both deionized water and ethanol. Multi-channel surface modified amorphous $\mathrm{Fe}_{2} \mathrm{O}_{3}$ nanospheres were obtained after drying in an oven at $110^{\circ} \mathrm{C}$ for $12 \mathrm{~h}$. Similarly, polycrystalline $\mathrm{Fe}_{2} \mathrm{O}_{3}$ nanospheres were prepared from the $\mathrm{FeCl}_{3}$ and $\mathrm{Ni}\left(\mathrm{CH}_{3} \mathrm{COO}\right)_{2}$ precursor solutions as a comparative sample (C1h).

\subsection{Characterization}

Crystallinity of the samples was examined by using X-ray diffraction (XRD) with a Bruker-D8 X-ray diffractometer with nickel filtered copper $\mathrm{K}$ radiation $(\lambda=1.5406 \AA)$. Morphologies of the samples were observed by using field emission scanning electron microscopy (FESEM, JEOL JSM-7500F) and transmission electron microscopy (TEM, JEM-2100P). Elemental compositions and chemical states were analyzed by using X-ray photoelectron spectroscopy (XPS, Thermo K-Alpha). Specific surface area of the samples was measured by using the Brutern-Emmett-Teller (BET) method with a Micrometrics ASAP 2420 surface analyzer.

\subsection{Electrochemical characterization}

The active materials (80 wt.\%) were mixed with conductive carbon black (10 wt.\%) and CMC (10 wt.\%) in deionized water to form slurries. The slurries were coated on nickel foam (99.5\%, Alfa Aesar) as current collector, followed by drying in a vacuum oven at $80^{\circ} \mathrm{C}$ for $12 \mathrm{~h}$ to obtain electrodes. Coin cells (CR 2032) with lithium foil as the counter electrode were assembled in a glove box filled with high-purity Ar gas (> 99.999\%). Electrolyte consisting of $1 \mathrm{M} \mathrm{LiPF}_{6}$ in a mixture of vinyl acetate (EC), ethylene carbonate (DEC) and dimethyl carbonate (DMC) (1:1:1 by volume) was used with a micro-porous polymer membrane separator (Celgard 2400). The cells were charged and discharged between $0.005 \mathrm{~V}$ and $3 \mathrm{~V}\left(\right.$ versus $\mathrm{Li}^{\mathrm{L}} \mathrm{Li}^{+}$) using blue electric test system (CT2001A). Galvanostatic intermittent titration technique (GITT) was employed by charging/discharging the cells at a current of $100 \mathrm{~mA} / \mathrm{g}$ for $20 \mathrm{~min}$ and it took about $4 \mathrm{~h}$ until the cut-off voltage limits were reached. Prior to post-cycling characterization, the cells were charged at $3 \mathrm{~V}$ for $48 \mathrm{~h}$ to ensure full extraction of Li. CHI660E electrochemical analyzer was used to record cyclic voltammetry (CV) and electrochemical impedance spectroscopy (EIS). The voltage range of CV measurement was $0.01-3 \mathrm{~V}$ and the scan rate was $0.1 \mathrm{mV} / \mathrm{s}$. The EIS measurement was conducted from $100 \mathrm{kHz}$ to $10 \mathrm{MHz}$. 


\section{Results And Discussion}

Figure 1 shows schematic diagrams describing formation process of the multi-channel surface modified amorphous $\mathrm{Fe}_{2} \mathrm{O}_{3}$ nanospheres during the hydrothermal treatment and the lattice evolution of $\mathrm{Fe}_{2} \mathrm{O}_{3}$ due to corrosion. Fig. 2 shows XRD patterns of the samples $\mathrm{A} 1 \mathrm{~h}$ and $\mathrm{C} 1 \mathrm{~h}$. The diffraction peaks of $\mathrm{C} 1 \mathrm{~h}$ are consistent with those of $\mathrm{a}-\mathrm{Fe}_{2} \mathrm{O}_{3}$ (No.33-0664), indicating that it is crystalline $\mathrm{a}-\mathrm{Fe}_{2} \mathrm{O}_{3}$. In contrast, $\mathrm{A} 1 \mathrm{~h}$ has no obvious diffraction peaks, suggesting that it is amorphous. As the hydrothermal treatment time was extended from $1 \mathrm{~h}$ to $6 \mathrm{~h}$, the XRD pattern of the samples remained unchanged (Fig. S1).

Fig. 2. XRD patterns of the samples A1h and C6h.

[IMAGE-C:\Workspace\ACDC\ImageHandler\4e

Figure 3 shows wide-scan XPS spectrum of A1h, confirming the presence of Fe and 0 in the amorphous sample. Fe 2p XPS spectrum is shown as an inset in Figure 3. The two peaks at $710.7 \mathrm{eV}$ and $724.5 \mathrm{eV}$ correspond to the binding energies of Fe $2 p 3 / 2$ and Fe $2 p 1 / 2$, respectively. The two peaks are consistent with the peaks of $\mathrm{Fe}^{3+}[39,40]$. In addition, the satellite peak at $719 \mathrm{eV}$ further confirms that the cation is $\mathrm{Fe}^{3+}$ [26]. Meanwhile, there is an obvious characteristic peak of lattice oxygen ( $01 \mathrm{~s}$ ) at $530 \mathrm{eV}$, as shown in Fig. S2 [41, 42]. Based on the XRD pattern in Fig. 2, it is concluded that amorphous $\mathrm{Fe}_{2} \mathrm{O}_{3}$ was formed in the samples $(\mathrm{A} 1 \mathrm{~h})$ prepared by using the hydrothermal reaction method with the precursor solution of $\mathrm{K}_{3}\left[\mathrm{Fe}(\mathrm{CN})_{6}\right]$ and $\mathrm{NH}_{4} \mathrm{H}_{2} \mathrm{PO}_{4}$.

Figure 3. XPS survey and Fe 2p spectra (inset) of the amorphous sample (A1h).

There are two main effects of $\mathrm{H}^{+}$ions on the structure and morphology of the synthesized $\mathrm{Fe}_{2} \mathrm{O}_{3}$. On the one hand, $\mathrm{H}^{+}$ions corrode crystal nuclei to form amorphous; On the other hand, $\mathrm{H}^{+}$ions corrode the surface of nanospheres into multiple staggered channels. In high temperature and high pressure environment in the hydrothermal kettle, hematite nuclei formed due to the hydrolysis of the $\mathrm{Fe}^{3+}$ ions in $\mathrm{K}_{3}\left[\mathrm{Fe}(\mathrm{CN})_{6}\right]$ were easily etched by $\mathrm{H}^{+}$ions from $\mathrm{NH}_{4} \mathrm{H}_{2} \mathrm{PO}_{4}[43]$. The nuclei lose the periodicity of their original crystal structure. In other words, the nuclei became amorphous, as shown in Fig. 1 (b), which then grew into amorphous nanoparticles. As the hydrothermal reaction continues, the amorphous nanoparticles converged into spheres to reduce the total surface energy. The surface of the spheres was smooth after reaction for $1 \mathrm{~h}$, as shown in Fig. 4 (a). Combined the XRD pattern (Fig. S1) and the electron diffraction (SAED) pattern (Fig. 4(g)) of the sample A1h, it could be determined that the sample A1h was amorphous. In addition, the high concentration of $\mathrm{H}^{+}$ions in the solution continues to corrode $\mathrm{Fe}_{2} \mathrm{O}_{3}$ nanospheres. A few holes appeared on the surface of nanoparticles after $3 \mathrm{~h}$ of hydrothermal reaction (Fig. 4 (b)). As the reaction time was increased to $6 \mathrm{~h}$, the pores on the surface of the nanospheres continue to be corroded and grow to form irregular interconnected channels (Fig. 4 (c)). To reveal surface characteristics of the nanospheres, $\mathrm{N}_{2}$ adsorption-desorption measurement were performed to obtain the BET surface area and the Barrett-Joyner-Halenda (BJH) pore size distribution profile, with the results to be shown in Fig. S3. The sample A3h has a pore size distribution in the range of 2-10 nm, while the surface 
of the A6h sample has multiple small holes that are connected to form channels ( $30-60 \mathrm{~nm})$. The results are consistent with the SEM observation. Representative TEM images of the samples reacted for different times are shown in Fig. $4(\mathrm{~d}-\mathrm{f})$, indicating hollow structure of the $\mathrm{Fe}_{2} \mathrm{O}_{3}$ nanospheres. With increasing hydrothermal treatment time, the wall thickness of the hollow nanospheres is decreased. This observation can be understood according to the Ostwald ripening process, because the inner particles have higher surface energy than the outer ones [43].

Electrochemical performances of the multi-channel surface modified amorphous $\mathrm{Fe}_{2} \mathrm{O}_{3}$ nanospheres and the crystalline $\mathrm{Fe}_{2} \mathrm{O}_{3}$ nanospheres were comparatively studied, in terms of cyclic voltammetry (CV) and galvanostatic charge/discharge curves. Fig. 5 shows CV curves of the two samples. CV curves of the crystalline $\mathrm{a}-\mathrm{Fe}_{2} \mathrm{O}_{3}$ nanospheres are similar to those previously reported in the literature [44, 45]. Comparatively, there are three differences in the $\mathrm{CV}$ curve between the amorphous and the crystalline $\mathrm{Fe}_{2} \mathrm{O}_{3}$ nanospheres. Firstly, the intensity of the reduction peak of the amorphous $\mathrm{Fe}_{2} \mathrm{O}_{3}$ during the first two cycles is lower than that of the crystalline $\mathrm{Fe}_{2} \mathrm{O}_{3}$. This may be attributed to the long-range disorder of the amorphous state and its low reaction Gibbs free energy change $(\Delta G)$, i.e., lithium can be intercalated/deintercalated more easily in amorphous $\mathrm{Fe}_{2} \mathrm{O}_{3}$. The volume change of the amorphous electrode is a gradual process, different from the sudden change of the crystalline electrode, which is beneficial to the integrity and cycle stability of the electrode $[26,46]$. Secondly, the main cathode peak shifted by $0.05 \mathrm{~V}$ in the second cathodic scan and the magnitude of the peak shift is much smaller than that of the crystalline $\mathrm{Fe}_{2} \mathrm{O}_{3}$, which may be caused by the better reversibility of the amorphous $\mathrm{Fe}_{2} \mathrm{O}_{3}$ electrode. The peak near 1.2-1.4 V may be related to the formation of solid solution compounds, owing to the insertion of $\mathrm{Li}^{+}$ions into the amorphous $\mathrm{Fe}_{2} \mathrm{O}_{3}[26,47]$. Finally, the first anodic scan of the amorphous electrode has two cathodic peaks at $1.47 \mathrm{~V}$ and $2.0 \mathrm{~V}$, corresponding to the oxidation of $\mathrm{Fe}(0)$ to $\mathrm{Fe}^{2+}$ and further to $\mathrm{Fe}^{3+}$, respectively $[47,48]$.

100nm(a)(b)100nm(c)100nm(d)(e)(f)(g)Fig. 4. SEM images of the samples: (a) A1h, (b) A3h and (c) A6h. TEM images of the samples: (d) A1h, (e) A3h and (f) A6h. (g) SAED pattern of A1h.

Figure 5. CV curves of the amorphous $\mathrm{Fe}_{2} \mathrm{O}_{3}$ nanospheres $\mathrm{A6h}$ (a) and crystalline a- $\mathrm{Fe}_{2} \mathrm{O}_{3}$ nanospheres C1h (b).

Galvanostatic charge/discharge measurements were conducted at a current density of $100 \mathrm{~mA} / \mathrm{g}$ in the voltage range of $0.005-3.0 \mathrm{~V}$. As shown in Fig. $6(\mathrm{a}, \mathrm{b})$, the first discharge specific capacity of the amorphous electrode (A6h) is $1187.3 \mathrm{mAh} / \mathrm{g}$. The value of the crystalline electrode (C1h) is slightly higher $(1305 \mathrm{mAh} / \mathrm{g})$. The large irreversible capacity of the two samples in the first cycle is a common phenomenon, which is related to the decomposition of electrolyte and the formation of SEl layer [48]. The specific capacity of the crystalline sample decreased rapidly, while the value of the amorphous one is much stable. The values are $817 \mathrm{mAh} / \mathrm{g}, 815.5 \mathrm{mAh} / \mathrm{g}, 818.8 \mathrm{mAh} / \mathrm{g}$, and $822.2 \mathrm{mAh} / \mathrm{g}$ in the four cycles. Fig. 6 (c) shows cycle performances of the amorphous and the crystalline $\mathrm{Fe}_{2} \mathrm{O}_{3}$ electrodes.

Comparatively, the amorphous electrode has higher cycle stability, with the specific capacity remaining at 
$875.2 \mathrm{mAh} / \mathrm{g}$ after 70 charge-discharge cycles. At the same time, the Coulombic efficiency is close to $100 \%$. Also, the cycle specific capacity is increased slightly, corresponding to a growth rate of $7.12 \%$. The increase in specific capacity of the amorphous electrode can be ascribed to the reversible formation of polymer gel-like film and the larger electrochemically active surface area of the $\mathrm{Fe}_{2} \mathrm{O}_{3}$ shell $[2,49,50]$. With further cycling, the capacitive-like storage effect is strengthened, which is advantageous for high power applications [51]. In addition, the amorphous $\mathrm{Fe}_{2} \mathrm{O}_{3}$ electrode (A6h) with multi-channel microstructure provided more active sites and space for lithium intercalation, thus promoting the interfacial lithium storage of the active materials, which also contributed to the high specific capacity [11, 52].

Figure 6 Discharge-charge voltage profiles of the amorphous and crystalline $\mathrm{Fe}_{2} \mathrm{O}_{3}$ electrodes for the initial five cycles at a rate of $100 \mathrm{~mA} / \mathrm{g}$ between 0.005 and $3.0 \mathrm{~V}$ : (a) A6h and (b) C1h. (c) Cycle performances of the A6h and $\mathrm{C} 1 \mathrm{~h}$ electrodes and their corresponding Coulombic efficiencies. (d) Rate performances of the $\mathrm{A} 6 \mathrm{~h}$ and $\mathrm{C} 1 \mathrm{~h}$ electrodes at different current rates. The 4th cycle charge/discharge profiles of amorphous and crystalline $\mathrm{Fe}_{2} \mathrm{O}_{3}$ electrodes at different current densities: (e) A6h and (f) C1h.

In order to further identify the difference in lithium ion storage performance between the amorphous and the crystalline $\mathrm{Fe}_{2} \mathrm{O}_{3}$ electrodes, rate performance tests were performed, with the results to be shown in Fig. 6 (d). When the current density is increased from 100 to $2000 \mathrm{~mA} / \mathrm{g}$, the average discharge capacities of the amorphous electrode (A6h) and the crystalline one (C1h) are decreased from 849.7 to $478.0 \mathrm{mAh} / \mathrm{g}$ and from 829.7 to $44.6 \mathrm{mAh} / \mathrm{g}$, respectively. As the current density is restored to $100 \mathrm{~mA} / \mathrm{g}$, the specific capacity of the amorphous electrode quickly recovered to $783.3 \mathrm{mAh} / \mathrm{g}$, which is much higher than that $(544.2 \mathrm{mAh} / \mathrm{g}$ ) of the crystalline electrode. The corresponding charge/discharge curves at different current densities are shown in Fig. 6 (e) and (f), respectively. As the current density is increased from 100 to $2000 \mathrm{~mA} / \mathrm{g}$, the discharge voltage plateau of the amorphous electrode (A6h) only slightly decreased, indicating that it has a relatively low polarization [53]. The outstanding rate performance is attributed to the well-distributed multi-channel structure, which offered a large electrode/electrolyte interface area and shortened the transport path of electrons and ions.

Notably, the discharge/charge voltage curves of amorphous and crystalline $\mathrm{Fe}_{2} \mathrm{O}_{3}$ electrodes during different cycles are shown in Fig. S4. During the cycle, the discharge platforms of the amorphous $\mathrm{Fe}_{2} \mathrm{O}_{3}$ electrodes are stable, indicating their low polarization and potential hysteresis[47]. The low potential hysteresis is linked with its faster kinetics and higher energy efficiency, which is an important factor for the practical applications [54]. In order to confirm this result and understand the ion diffusion kinetics, galvanostatic intermittent titration (GITT) measurement was utilized to analyze the lithium ion transport kinetics of the electrode. Fig. S5 shows potential change of the sample as a function of time. The cells were repeatedly subject to a current pulse of $100 \mathrm{~mA} / \mathrm{g}$ for $20 \mathrm{~min}$ and then relaxed for $240 \mathrm{~min}$. The long relaxation time was used to full relaxation of lithium diffusion to reach equilibrium potential and minimize the self-discharge of $\mathrm{Fe}_{2} \mathrm{O}_{3}$ during the test. The discharge/charge curves of the amorphous and crystalline electrodes have similar trends. Current pulse step polarization curves of the amorphous and 
the crystalline $\mathrm{Fe}_{2} \mathrm{O}_{3}$ electrodes at different potentials are shown in Fig. 7 (a) and (b), respectively. According to the polarization curves, when the cells went to a higher voltage upon charging, it took more time to relax the pulse to reach a stable state (Fig. 7 (a-2) and Fig. 7 (b-6)). Similarly, when the cells were discharged at a lower voltage, the pulse relaxation would be delayed (Fig. 7 (a-3) and Fig. 7 (b-7)). These phenomena indicate that the lithium diffusion coefficient will change with the change of potential. Overpotential refers to the voltage difference between the equilibrium potential at the end of relaxation and that at the end of the current pulse $\left(\approx \Delta \mathrm{E}_{\mathrm{T}}-\Delta \mathrm{E}_{\mathrm{s}}\right.$, ignoring IR drop) [55]. The overpotential during charging is greater than that during discharging. As clearly seen in Fig. 7 (a) and (b), the voltage hysteresis of the amorphous electrode is significantly less pronounced than that of the crystalline electrode. The difference in polarization may be attributed to their difference in kinetics [56].

In order to better understand diffusion kinetics of $\mathrm{Li}^{+}$, galvanostatic intermittent titration (GITT) data were used to derive the diffusion coefficients $\left(D_{\mathrm{Li}+}, \mathrm{cm}^{2} / \mathrm{s}\right)$ of the $\mathrm{Fe}_{2} \mathrm{O}_{3}$ electrodes, which can be estimated by using the Fick's law [51]:

$$
D_{L^{+}}=\frac{4}{\Pi \tau}\left(\frac{m_{b} V_{M}}{M_{b} S}\right)^{2}\left(\frac{\Delta E_{S}}{\Delta E_{\tau}}\right)^{2}
$$

where $\tau$ is the constant current pulse time; $m_{b}, V_{M}, M_{b}$ are the mass loading, molecular weight, molar volume of the active material, respectively, $S$ is the effective area between the electrode and electrolyte, $\Delta \mathrm{E}_{\mathrm{S}}$ and $\Delta \mathrm{E}_{\mathrm{T}}$ represent the change of steady-state voltage and the total change of cell voltage during pulse subtracting the IR drop. $\mathrm{D}_{\mathrm{Li}}$ values of the amorphous and the crystalline $\mathrm{Fe}_{2} \mathrm{O}_{3}$ electrodes are shown in Fig. 7 (c) and (d), respectively. It can be seen that the $D_{\mathrm{Li}}$ values in the two electrodes range from $10^{13}$ to $10^{10} \mathrm{~cm}^{2} / \mathrm{s}$, in agreement with the reported data of $\mathrm{a}-\mathrm{Fe}_{2} \mathrm{O}_{3}$ nanoparticles $\left(10^{14}-10^{11} \mathrm{~cm}^{2} / \mathrm{s}\right)$ [57] and $₫-\mathrm{Fe}_{2} \mathrm{O}_{3}$ electrode $\left(9.96 \times 10^{13} \mathrm{~cm}^{2} / \mathrm{s}\right)$ [58]. It is worth noting that the amorphous electrode showed higher $\mathrm{D}_{\mathrm{Li}+}$, because mainly of the lack of grain boundaries, thus shortening the diffusion pathways and reducing the diffusion resistance.

(a)(b)Fig. 7. GITT profiles of the amorphous and crystalline $\mathrm{Fe}_{2} \mathrm{O}_{3}$ electrodes: (a) A6h and (b) C1h. The insets show polarized curves for current pulse steps derived from the GITT data at different potentials in the discharge and charge processes. (c, d) $\mathrm{Li}^{+}$diffusion coefficients of the electrodes during the discharge/charge process at various voltages derived from the GITT data: (a) A6h and (b) C1h.

Reaction kinetics of the amorphous and the crystalline $\mathrm{Fe}_{2} \mathrm{O}_{3}$ electrodes were also evaluated by using electrochemical impedance spectroscopy (EIS). All Nyquist plots exhibited a recessed semicircle in the high-frequency region and a sloping line in the low-frequency region, which correspond to the charge transfer resistance and the diffusion impedance of $\mathrm{Li}^{+}$, respectively. As shown in Fig. 8, the semicircular 
diameter of the amorphous $\mathrm{Fe}_{2} \mathrm{O}_{3}$ electrode in the high frequency region is much smaller than that of the crystalline $\mathrm{Fe}_{2} \mathrm{O}_{3}$ electrode. Therefore, due to the noncrystalline nature, amorphous $\mathrm{Fe}_{2} \mathrm{O}_{3}$ greatly ensured a rapid charge transfer, thus facilitating faster lithiation/delithiation kinetics [38]. The EIS results are consistent with the cycle performances (Fig. 6 (c)) and the rate performances (Fig. 6 (d)).

\section{Conclusions}

In summary, multi-channel surface modified amorphous $\mathrm{Fe}_{2} \mathrm{O}_{3}$ nanospheres have been successfully prepared by using a facile hydrothermal method, due to the synergistic effect of ripening and hydrogen ion etching. Owing to the isotropic nature and the lack of grain boundaries, the amorphous $\mathrm{Fe}_{2} \mathrm{O}_{3}$ facilitated high lithium ion insertion and withstood high strains. The multi-channel surface modified structure of the amorphous $\mathrm{Fe}_{2} \mathrm{O}_{3}$ not only ensured close contact between the internal active materials and the electrolyte, but also effectively alleviated the volume change during the intercalation/deintercalation of lithium ions. The multi-channel surface modified amorphous $\mathrm{Fe}_{2} \mathrm{O}_{3}$ nanospheres electrode exhibited excellent cycle stability $(875.2 \mathrm{mAh} / \mathrm{g}$ after 70 cycles at $100 \mathrm{~mA} / \mathrm{g})$ and superior rate performance $(56.3 \%$ capacity retention from 0.1 to $2.0 \mathrm{~A} / \mathrm{g}$ ) and narrow voltage hysteresis. The amorphous $\mathrm{Fe}_{2} \mathrm{O}_{3}$ electrode exhibited faster electrochemical reaction kinetics, higher $\mathrm{Li}^{+}$diffusion coefficient and lower overpotential, as compared with its crystalline counterpart. The results in our present study can be used as a reference for the synthesis of amorphous transitional metal oxides (TMOs) as anodes of LIBs for practical applications.

\section{Declarations}

\section{Acknowledgments}

This work was financially supported by National Natural Science Regional Fund (No. 62041107, 61764007), the Natural Science Foundation of Jiangxi Province (No. 20181BAB202005), and the Education Bureau of Jiangxi Province. Shenzhen Technology University (SZTU) was acknowledged for the financial support of Start-up Grant (2018) and the Natural Science Foundation of Top Talent of SZTU (Grant No. 2019010801002).

\section{References}

[1] M.H. Ryou, J. Kim, I. Lee, S. Kim, Y.K. Jeong, S. Hong, J.H. Ryu, T.S. Kim, J.K. Park, H. Lee, J.W. Choi, Mussel-inspired adhesive binders for high-performance silicon nanoparticle anodes in lithium-ion batteries, Adv Mater, 25 (2013) 1571-1576.

[2] L. Liu, X. Huang, Z. Wei, X. Duan, B. Zhong, L. Xia, T. Zhang, H. Wang, D. Jia, Y. Zhou, R. Zhang, Solvents adjusted pure phase $\mathrm{CoCO} 3$ as anodes for high cycle stability, Journal of Advanced Ceramics, 10 (2021) 509-519. 
[3] Y. Zhao, J. Wang, C. Ma, L. Cao, Z. Shao, A self-adhesive graphene nanoscroll/nanosheet paper with confined Fe1-xS/Fe304 hetero-nanoparticles for high-performance anode material of flexible Li-ion batteries, Chemical Engineering Journal, 370 (2019) 536-546.

[4] S. Wang, Y. Yang, Y. Dong, Z. Zhang, Z. Tang, Recent progress in Ti-based nanocomposite anodes for lithium ion batteries, Journal of Advanced Ceramics, 8 (2019) 1-18.

[5] Q. Pan, P. Zuo, T. Mu, C. Du, X. Cheng, Y. Ma, Y. Gao, G. Yin, Improved electrochemical performance of micro-sized SiO-based composite anode by prelithiation of stabilized lithium metal powder, Journal of Power Sources, 347 (2017) 170-177.

[6] P. Wu, B. Shi, H. Tu, C. Guo, A. Liu, G. Yan, Z. Yu, Pomegranate-type Si/C anode with SiC taped, welldispersed tiny Si particles for lithium-ion batteries, Journal of Advanced Ceramics, 10 (2021) 1-11.

[7] M. Wu, Y. He, L. Wang, Q. Xia, A. Zhou, Synthesis and electrochemical properties of V2C MXene by etching in opened/closed environments, Journal of Advanced Ceramics, 9 (2020) 749-758.

[8] Y. Dong, B. Wang, K. Zhao, Y. Yu, X. Wang, L. Mai, S. Jin, Air-Stable Porous Fe2N Encapsulated in Carbon Microboxes with High Volumetric Lithium Storage Capacity and a Long Cycle Life, Nano Letters, 17 (2017) 5740-5746.

[9] H. Huang, S. Gao, A.-M. Wu, K. Cheng, X.-N. Li, X.-X. Gao, J.-J. Zhao, X.-L. Dong, G.-Z. Cao, Fe 3 N constrained inside $\mathrm{C}$ nanocages as an anode for Li-ion batteries through post-synthesis nitridation, Nano Energy, 31 (2017) 74-83.

[10] H. Li, In-situ Grown Hierarchical MoS2 Nanoflakes on Three- Dimensional Carbon Fiber Papers as Free-Standing Anodes for Lithium-Ion Battery, International Journal of Electrochemical Science, (2019) 8662-8675.

[11] Y. Zhao, X. Li, B. Yan, D. Xiong, D. Li, S. Lawes, X. Sun, Recent Developments and Understanding of Novel Mixed Transition-Metal Oxides as Anodes in Lithium Ion Batteries, Advanced Energy Materials, 6 (2016) 1502175.

[12] S. Wang, B. Tang, W. Yang, F. Wu, G. Zhang, B. Zhao, X. He, Y. Yang, J. Jiang, The flower-like heterostructured Fe203/MoS2 coated by amorphous Si-Oxyhydroxides: An effective surface modification method for sulfide photocatalysts in photo-Fenton reaction, Journal of Alloys and Compounds, 784 (2019) 1099-1105.

[13] S. Yu, V.M. Hong Ng, F. Wang, Z. Xiao, C. Li, L.B. Kong, W. Que, K. Zhou, Synthesis and application of iron-based nanomaterials as anodes of lithium-ion batteries and supercapacitors, Journal of Materials Chemistry A, 6 (2018) 9332-9367.

[14] Z. Ren, S. Yu, B. Han, Z. Shao, Z. Wang, One-pot carbonization synthesis of $Y$-Fe203/Fe/carbon composite for high Li-storage and excellent stability, Materials Letters, 275 (2020) 128066. 
[15] Y. Jin, Dang, Liyun,Zhang, Hao,Song, Chuang,Lu, Qingyi,Gao, Feng, Synthesis of unit-cell-thick a-Fe 2 03 nanosheets and their transformation to $y-F e ~ 203$ nanosheets with enhanced LIB performances, Chemical Engineering Journal, 326 (2017) 292-297.

[16] G. Binitha, Soumya, M. S., Madhavan, Asha Anish, Praveen, P., Balakrishnan, A., Subramanian, K. R. V., Reddy, M. V., Nair, Shantikumar V., Nair, A. Sreekumaran, Sivakumar, N., Electrospun a-Fe2O3 nanostructures for supercapacitor applications, Journal of Materials Chemistry A, 1 (2013) 11698.

[17] Y. Park, Oh, Misol,Park, Jung Soo,Baek, Seong-Ho,Kim, Minsun,Kim, Soonhyun,Kim, Jae Hyun, Electrochemically deposited Fe2O3 nanorods on carbon nanofibers for free-standing anodes of lithiumion batteries, Carbon, 94 (2015) 9-17.

[18] C. Gu, Song, Xinjie, Zhang, Simin, Ryu, Si Ok, Huang, Jiarui, Synthesis of hierarchical a-Fe 203 nanotubes for high-performance lithium-ion batteries, Journal of Alloys and Compounds, 714 (2017) 612.

[19] J. Lu, Peng, Qing, Wang, Zhongying, Nan, Caiyun, Li, Lihong, Li, Yadong, Hematite nanodiscs exposing (001) facets: synthesis, formation mechanism and application for Li-ion batteries, Journal of Materials Chemistry A, 1 (2013) 5232.

[20] L. Zhang, Wu, H. B., Madhavi, S., Hng, H. H., Lou, X. W., Formation of Fe2O3 microboxes with hierarchical shell structures from metal-organic frameworks and their lithium storage properties, $\mathrm{J}$ Am Chem Soc, 134 (2012) 17388-17391.

[21] K. Cao, Jiao, Lifang,Liu, Huiqiao,Liu, Yongchang,Wang, Yijing,Guo, Zaiping,Yuan, Huatang, 3D Hierarchical Porous a-Fe203Nanosheets for High-Performance Lithium-lon Batteries, Advanced Energy Materials, 5 (2015) 1401421.

[22] J.S. Cho, Hong, Y. J., Lee, J. H., Kang, Y. C., Design and synthesis of micron-sized spherical aggregates composed of hollow Fe2O3 nanospheres for use in lithium-ion batteries, Nanoscale, 7 (2015) 8361-8367.

[23] X. Qi, Zhang, H. B., Xu, J., Wu, X., Yang, D., Qu, J., Yu, Z. Z., Highly Efficient High-Pressure Homogenization Approach for Scalable Production of High-Quality Graphene Sheets and SandwichStructured alpha-Fe203/Graphene Hybrids for High-Performance Lithium-lon Batteries, ACS Appl Mater Interfaces, 9 (2017) 11025-11034.

[24] H. Zhou, M. Jin, B. Zhou, J. Zhao, W. Han, Porous nanotube networks of Sn02/Mo03@Graphene as anodes for rechargeable lithium-ion batteries, Nanotechnology, 32 (2021) 095704.

[25] Y. Zhang, J. Yang, Y. Zhang, C. Li, W. Huang, Q. Yan, X. Dong, Fe2O3/SnSSe Hexagonal Nanoplates as Lithium-Ion Batteries Anode, ACS Appl Mater Interfaces, 10 (2018) 12722-12730. 
[26] Y. Jiang, D. Zhang, Y. Li, T. Yuan, N. Bahlawane, C. Liang, W. Sun, Y. Lu, M. Yan, Amorphous Fe2O3 as a high-capacity, high-rate and long-life anode material for lithium ion batteries, Nano Energy, 4 (2014) 2330 .

[27] X. Zhu, X. Jiang, X. Chen, X. Liu, L. Xiao, Y. Cao, Fe 203 amorphous nanoparticles/graphene composite as high-performance anode materials for lithium-ion batteries, Journal of Alloys and Compounds, 711 (2017) 15-21.

[28] L. Shi, Y. Li, F. Zeng, S. Ran, C. Dong, S.-Y. Leu, S.T. Boles, K.H. Lam, In situ growth of amorphous Fe2O3 on 3D interconnected nitrogen-doped carbon nanofibers as high-performance anode materials for sodium-ion batteries, Chemical Engineering Journal, 356 (2019) 107-116.

[29] Z. Wang, Z. Wang, W. Liu, W. Xiao, X.W. Lou, Amorphous CoSn03@C nanoboxes with superior lithium storage capability, Energy \& Environmental Science, 6 (2013) 87-91.

[30] P. Heitjans, E. Tobschall, M. Wilkening, lon transport and diffusion in nanocrystalline and glassy ceramics, The European Physical Journal Special Topics, 161 (2008) 97-108.

[31] U. Dash, S. Sahoo, S.K.S. Parashar, P. Chaudhuri, Effect of Li+ ion mobility on the grain boundary conductivity of Li2TiO3 nanoceramics, Journal of Advanced Ceramics, 3 (2014) 98-108.

[32] E. Hüger, L. Dörrer, J. Rahn, T. Panzner, J. Stahn, G. Lilienkamp, H. Schmidt, Lithium Transport through Nanosized Amorphous Silicon Layers, Nano Letters, 13 (2013) 1237-1244.

[33] X. Li, X. Meng, J. Liu, D. Geng, Y. Zhang, M.N. Banis, Y. Li, J. Yang, R. Li, X. Sun, M. Cai, M.W. Verbrugge, Tin Oxide with Controlled Morphology and Crystallinity by Atomic Layer Deposition onto Graphene Nanosheets for Enhanced Lithium Storage, Advanced Functional Materials, 22 (2012) 16471654.

[34] M.T. McDowell, S.W. Lee, J.T. Harris, B.A. Korgel, C. Wang, W.D. Nix, Y. Cui, In Situ TEM of Two-Phase Lithiation of Amorphous Silicon Nanospheres, Nano Letters, 13 (2013) 758-764.

[35] H. Xiong, M.D. Slater, M. Balasubramanian, C.S. Johnson, T. Rajh, Amorphous TiO2 Nanotube Anode for Rechargeable Sodium Ion Batteries, The Journal of Physical Chemistry Letters, 2 (2011) 2560-2565.

[36] P. Peng, Q. Zhao, P. Zhu, W. Liu, Y. Yuan, R. Ding, P. Gao, X. Sun, E. Liu, Amorphous Fe2O3 film-coated mesoporous Fe2O3 core-shell nanosphere prepared by quenching as a high-performance anode material for lithium-ion batteries, Journal of Electroanalytical Chemistry, 898 (2021) 115633.

[37] O. Delmer, P. Balaya, L. Kienle, J. Maier, Enhanced Potential of Amorphous Electrode Materials: Case Study of RuO2, Advanced Materials, 20 (2008) 501-505.

[38] J. Guo, Q. Liu, C. Wang, M.R. Zachariah, Interdispersed Amorphous MnOx-Carbon Nanocomposites with Superior Electrochemical Performance as Lithium-Storage Material, Advanced Functional Materials, 
$22(2012)$ 803-811.

[39] J.C.Y.a.J.G. Xianluo Hu, Fast Production of Self-Assembled Hierarchical r-Fe2O3 Nanoarchitectures, Journal of Physical Chemistry C, 111 (2007) 11180-11185.

[40] H.T. Yizhi Yan, Fan Wu, Rui Wang, Mu Pan, One-Step Self-Assembly Synthesis a-Fe2O3 with CarbonCoated Nanoparticles for Stabilized and Enhanced Supercapacitors Electrode, Energies, 10 (2017) 1296.

[41] L.-C. Hsu, Y.-Y. Li, C.-G. Lo, C.-W. Huang, G. Chern, Thermal growth and magnetic characterization of aFe203nanowires, Journal of Physics D: Applied Physics, 41 (2008).

[42] S. Sun, T. Zhai, C. Liang, S.V. Savilov, H. Xia, Boosted crystalline/amorphous Fe203-ס core/shell heterostructure for flexible solid-state pseudocapacitors in large scale, Nano Energy, 45 (2018) 390-397.

[43] P. Sun, B. Wang, L. Zhao, H. Gao, T. Wang, X. Yang, C. Liu, G. Lu, Enhanced gas sensing by amorphous double-shell Fe 203 hollow nanospheres functionalized with PdO nanoparticles, Sensors and Actuators B: Chemical, 252 (2017) 322-329.

[44] J. Zhu, Z. Yin, D. Yang, T. Sun, H. Yu, H.E. Hoster, H.H. Hng, H. Zhang, Q. Yan, Hierarchical hollow spheres composed of ultrathin Fe2O3 nanosheets for lithium storage and photocatalytic water oxidation, Energy \& Environmental Science, 6 (2013) 987-993.

[45] X. Lv, J. Deng, J. Wang, J. Zhong, X. Sun, Carbon-coated a-Fe2O3 nanostructures for efficient anode of Li-ion battery, Journal of Materials Chemistry A, 3 (2015) 5183-5188.

[46] M.V. Reddy, T. Yu, C.H. Sow, Z.X. Shen, C.T. Lim, G.V. Subba Rao, B.V.R. Chowdari, a-Fe2O3 Nanoflakes as an Anode Material for Li-lon Batteries, Advanced Functional Materials, 17 (2007) 2792-2799.

[47] Y. Huang, Z. Lin, M. Zheng, T. Wang, J. Yang, F. Yuan, X. Lu, L. Liu, D. Sun, Amorphous Fe 203 nanoshells coated on carbonized bacterial cellulose nanofibers as a flexible anode for high-performance lithium ion batteries, Journal of Power Sources, 307 (2016) 649-656.

[48] B. Wang, J.S. Chen, H.B. Wu, Z. Wang, X.W. Lou, Quasiemulsion-templated formation of alpha-Fe2O3 hollow spheres with enhanced lithium storage properties, J Am Chem Soc, 133 (2011) 17146-17148.

[49] X. Jiang, X. Yang, Y. Zhu, Y. Yao, P. Zhao, C. Li, Graphene/carbon-coated Fe304 nanoparticle hybrids for enhanced lithium storage, Journal of Materials Chemistry A, 3 (2015) 2361-2369.

[50] X. Wang, X.-L. Wu, Y.-G. Guo, Y. Zhong, X. Cao, Y. Ma, J. Yao, Synthesis and Lithium Storage Properties of Co304 Nanosheet-Assembled Multishelled Hollow Spheres, Advanced Functional Materials, 20 (2010) 1680-1686.

[51] B. Sun, S. Lou, Z. Qian, P. Zuo, C. Du, Y. Ma, H. Huo, J. Xie, J. Wang, G. Yin, Pseudocapacitive Li+ storage boosts ultrahigh rate performance of structure-tailored CoFe2O4@Fe2O3 hollow spheres 
triggered by engineered surface and near-surface reactions, Nano Energy, 66 (2019) 104179.

[52] R. Jin, Y. Ma, Y. Sun, H. Li, Q. Wang, G. Chen, Manganese Cobalt Oxide (MnCo204) Hollow Spheres as High Capacity Anode Materials for Lithium-Ion Batteries, Energy Technology, 5 (2017) 293-299.

[53] J. Xin, C. Jia-jia, X. Jian-hui, S. Yi-ning, F. You-zuo, Z. Min-sen, D. Quan-feng, Fe2O3 xerogel used as the anode material for lithium ion batteries with excellent electrochemical performance, Chem Commun (Camb), 48 (2012) 7410-7412.

[54] V. Etacheri, R. Marom, R. Elazari, G. Salitra, D. Aurbach, Challenges in the development of advanced Li-ion batteries: a review, Energy \& Environmental Science, 4 (2011) 3243-3262.

[55] Y. Zhu, C. Wang, Strain accommodation and potential hysteresis of LiFePO4 cathodes during lithium ion insertion/extraction, Journal of Power Sources, 196 (2011) 1442-1448.

[56] Y. Li, J. Ji, J. Yao, Y. Zhang, B. Huang, G. Cao, Sodium ion storage performance and mechanism in orthorhombic V2O5 single-crystalline nanowires, Science China Materials, 64 (2020) 557-570.

[57] J. Yao, Y. Yang, Y. Li, J. Jiang, S. Xiao, J. Yang, Interconnected a-Fe2O3 nanoparticles prepared from leaching liquor of tin ore tailings as anode materials for lithium-ion batteries, Journal of Alloys and Compounds, 855 (2021) 157288.

[58] C. Wu, Y. Xu, L. Ao, K. Jiang, L. Shang, Y. Li, Z. Hu, J. Chu, Robust three-dimensional porous rGO aerogel anchored with ultra-fine a-Fe2O3 nanoparticles exhibit dominated pseudocapacitance behavior for superior lithium storage, Journal of Alloys and Compounds, 816 (2020) 152627.

\section{Figures}

\section{Figure 1}

(a) Schematic diagram of the formation of the multi-channel surface modified amorphous $\mathrm{Fe} 2 \mathrm{O} 3$ nanospheres during the hydrothermal reaction process. (b) Schematic diagram of the evolution of Fe2O3 lattice due to corrosion. 


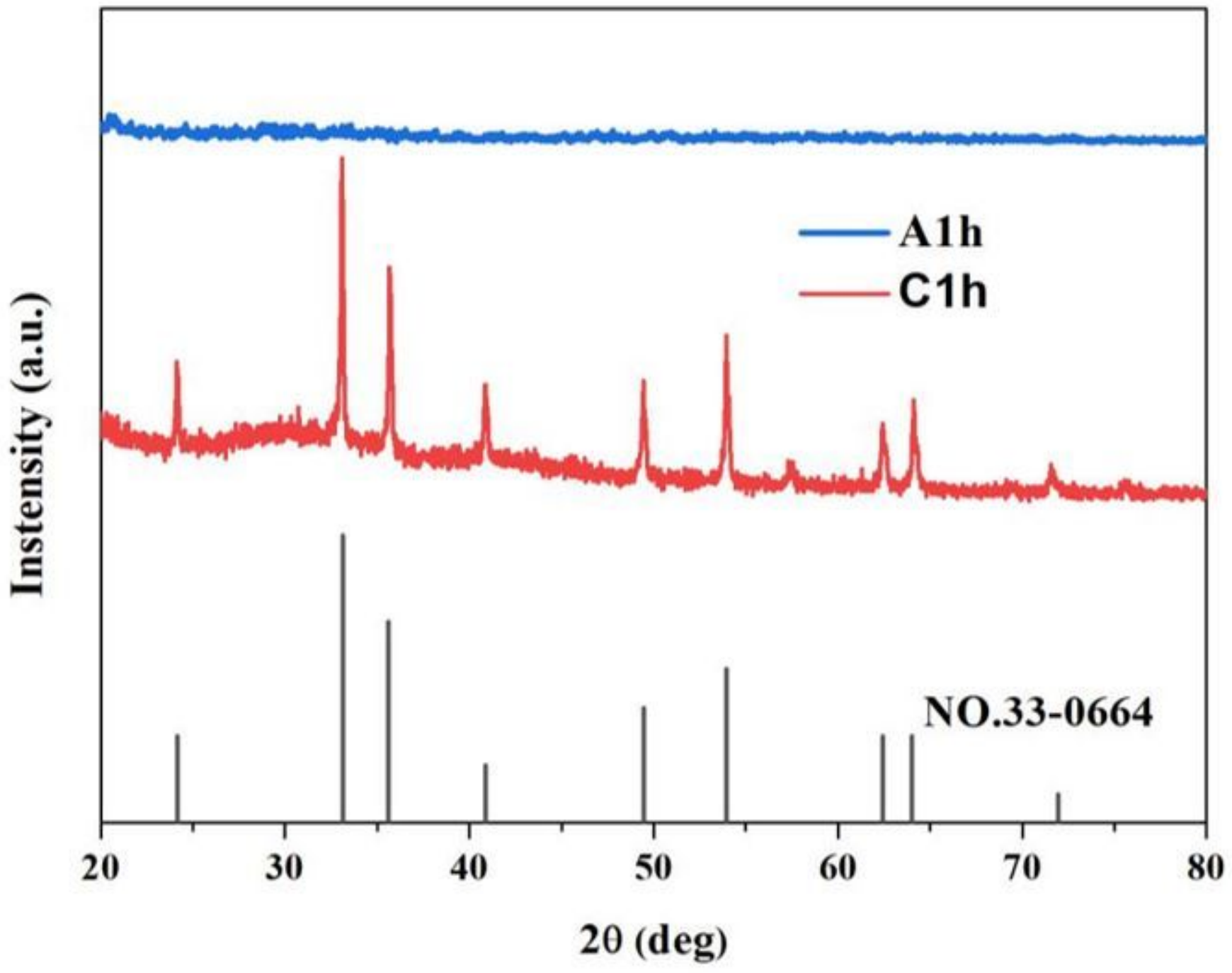

Figure 2

XRD patterns of the samples A1h and C6h. 


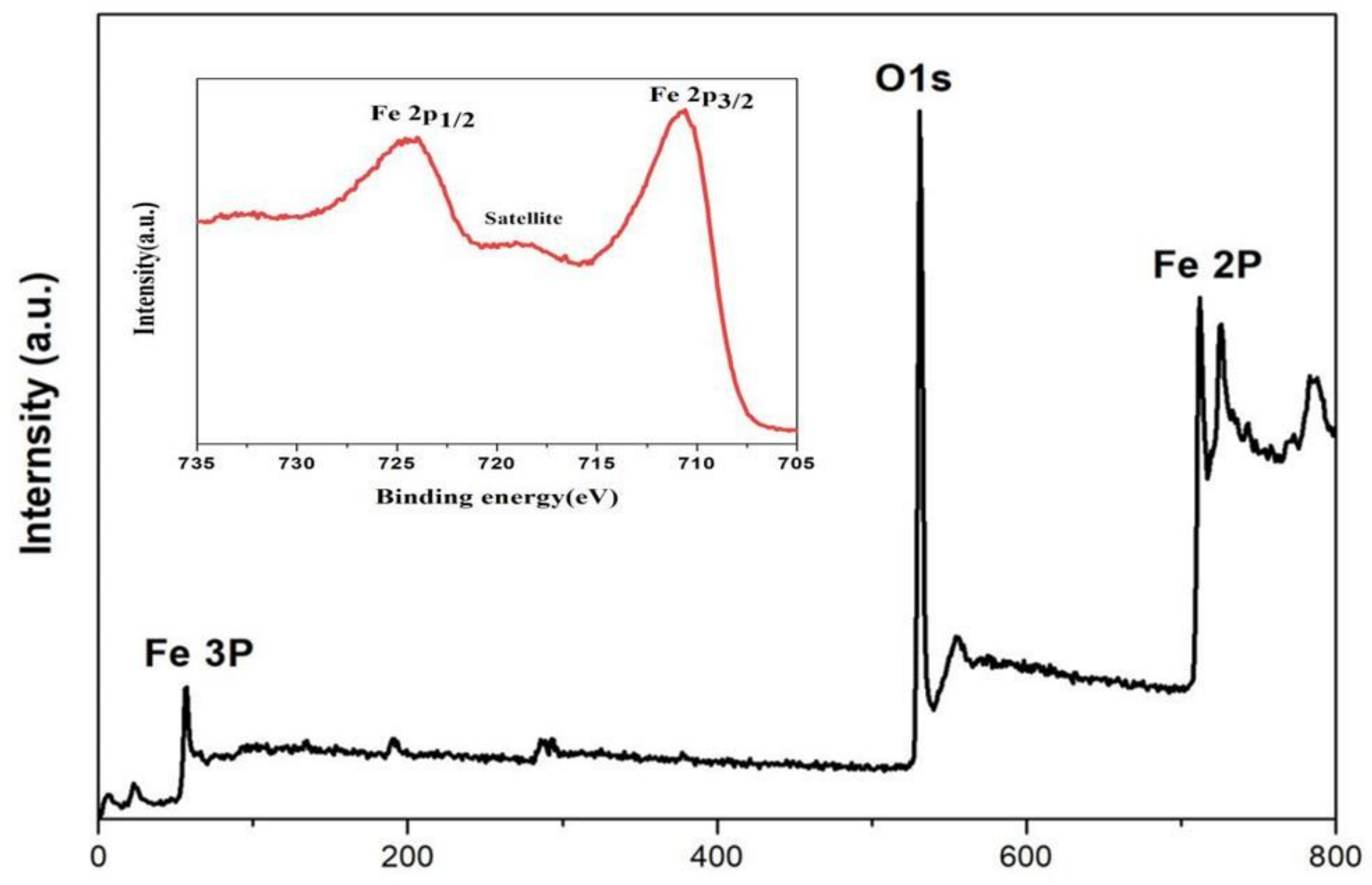

Figure 3

XPS survey and Fe 2p spectra (inset) of the amorphous sample (A1h). 

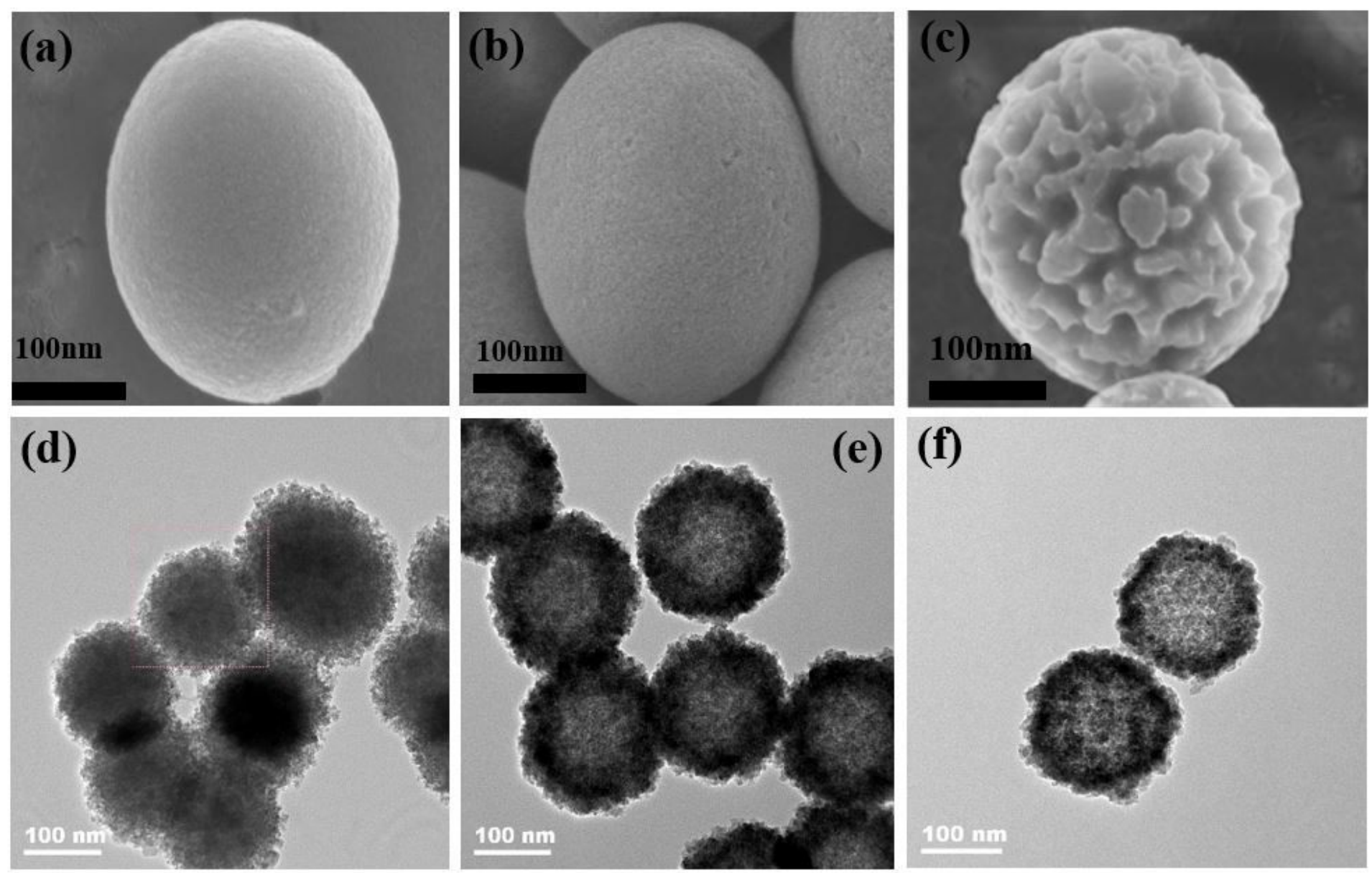

(f)

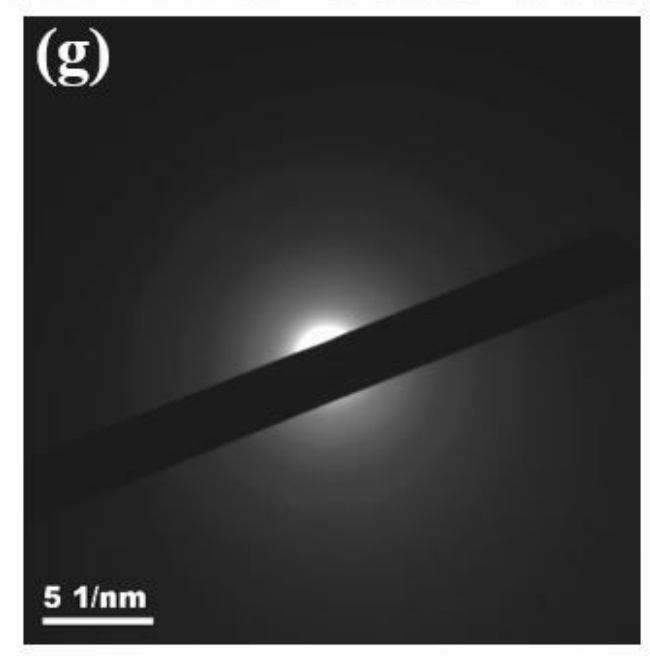

$100 \mathrm{~nm}$

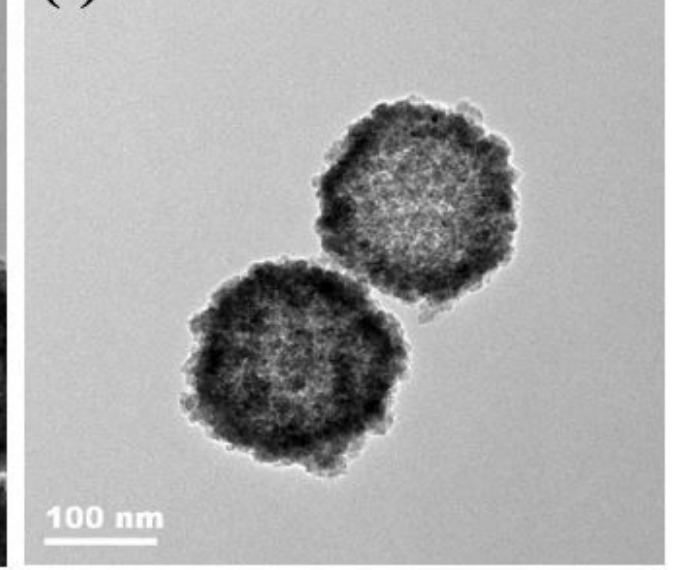

(1000,

\section{Figure 4}

SEM images of the samples: (a) A1h, (b) A3h and (c) A6h. TEM images of the samples: (d) A1h, (e) A3h and (f) A6h. (g) SAED pattern of A1h. 

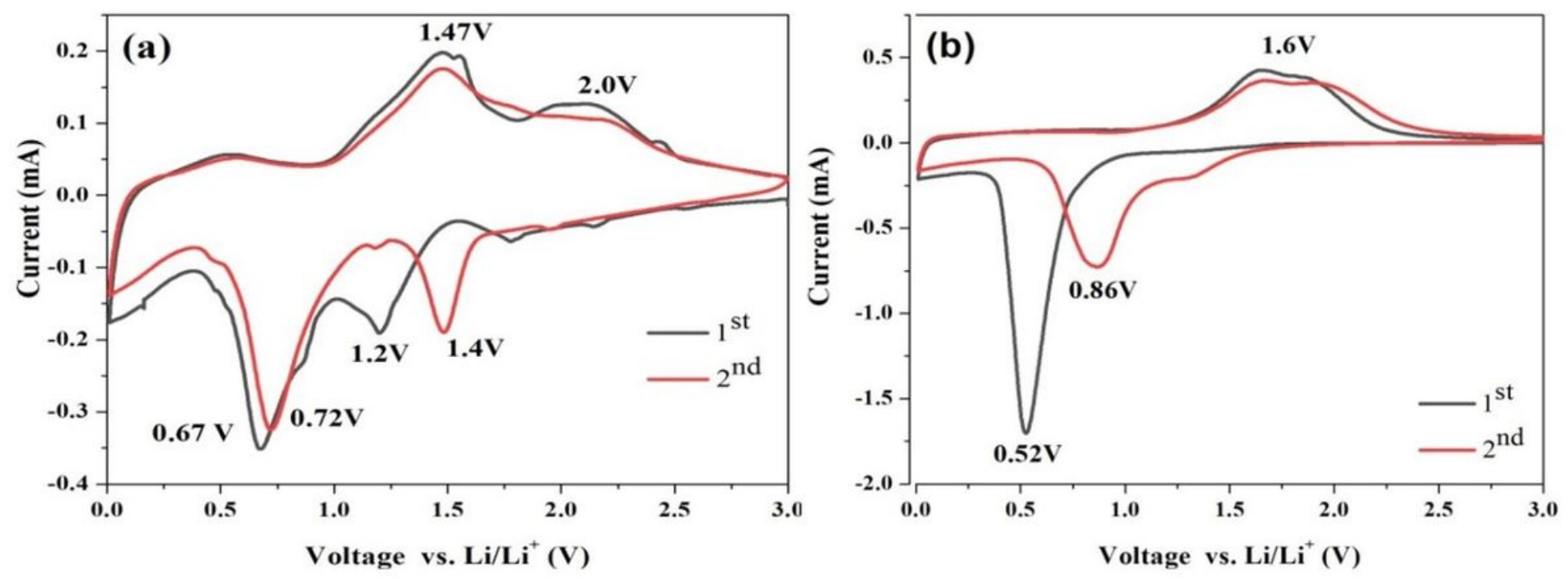

Figure 5

CV curves of the amorphous Fe2O3 nanospheres A6h (a) and crystalline a-Fe2O3 nanospheres C1h (b).

\section{Figure 6}

Discharge-charge voltage profiles of the amorphous and crystalline Fe2O3 electrodes for the initial five cycles at a rate of $100 \mathrm{~mA} / \mathrm{g}$ between 0.005 and $3.0 \mathrm{~V}$ : (a) A6h and (b) C1h. (c) Cycle performances of the A6 h and $\mathrm{C} 1 \mathrm{~h}$ electrodes and their corresponding Coulombic efficiencies. (d) Rate performances of the $\mathrm{A} 6 \mathrm{~h}$ and $\mathrm{C} 1 \mathrm{~h}$ electrodes at different current rates. The 4th cycle charge/discharge profiles of amorphous and crystalline Fe2O3 electrodes at different current densities: (e) A6h and (f) C1h.

\section{Figure 7}

GITT profiles of the amorphous and crystalline Fe203 electrodes: (a) A6h and (b) C1h. The insets show polarized curves for current pulse steps derived from the GITT data at different potentials in the discharge and charge processes. (c, d) Li+ diffusion coefficients of the electrodes during the discharge/charge process at various voltages derived from the GITT data: (a) A6h and (b) C1h.

\section{Figure 8}

Nyquist plots of the amorphous and the crystalline Fe2O3 electrodes.

\section{Supplementary Files}


This is a list of supplementary files associated with this preprint. Click to download.

- SupplementaryMaterial.docx 